\title{
Educación, género y división del trabajo. Articulaciones en el marco de las "políticas de igualdad de oportunidades" en Argentina (1991-1994)
}

\author{
Education, gender and division of labor. Articulations within \\ the framework of "equal opportunities policies" In Argentina \\ (1991-1994)
}

Matías Alvarez*

Florencia Minardo**

\begin{abstract}
Resumen
El presente trabajo se enmarca en el proyecto de incentivos denominado "Estado, políticas públicas y desigualdades sexogenéricas. Configuraciones en la Argentina contemporánea (1983-2019)", el cual pretende dar cuenta de las formas de intervención estatal implicadas en la reproducción/ transformación de estas desigualdades y su relación con los procesos sociales y culturales más amplios. Uno de los objetivos de este proyecto es el análisis de las políticas de género que se han institucionalizado progresivamente desde el retorno a la democracia, y las tensiones a las que han dado lugar. Aquí nos detendremos particularmente en el trabajo como una dimensión de la desigualdad de género identificada como central en estas iniciativas políticas, y el recurso a la educación y las políticas educativas, como instrumentos transformar esta dimensión de la desigualdad. A tal efecto, buscaremos identificar cómo la división sexual del trabajo ha sido colocada como un vector central de intervención social en distintos programas e iniciativas desplegadas por los organismos para la mujer, especialmente durante la primera gestión del Consejo Nacional de la Mujer. Luego, intentaremos mostrar las

\footnotetext{
* Universidad Nacional del Centro de la Provincia de Buenos Aires. Instituto de Geografía, Historia y Ciencias Sociales- Grupo Interdisciplinario en Ciencia Sociedad y Cultura/ Consejo Nacional de Investigaciones Científicas y Técnicas (UNICEN- IGEHCS-Ci.So.C). Mail: alvarezmatias@gmail.com.

** Universidad Nacional del Centro de la Provincia de Buenos Aires. Instituto de Geografía, Historia y Ciencias Sociales- Grupo Interdisciplinario en Ciencia Sociedad y Cultura/ Consejo Nacional de Investigaciones Científicas y Técnicas (UNICEN- IGEHCS-Ci.So.C). Mail: minardoflorencia@gmail.com.
} 
respuestas educativas al problema de la división sexual del trabajo desarrolladas por el Consejo en el mismo período en un contexto de reformas educativas.

Palabras claves: Políticas públicas, desigualdad de género, trabajo, educación.

\begin{abstract}
This work is part of the incentive project called "State, public policies and sex-gender inequalities. Configurations in contemporary Argentina (1983-2019) "which aims to account for the forms of state intervention involved in the reproduction / transformation of these inequalities and their relationship with broader social and cultural processes. One of the objectives of this project is the analysis of gender policies that have been progressively institutionalized since the return to democracy, and the tensions to which they have given rise. Here we will dwell particularly on work as a dimension of gender inequality identified as central to these political initiatives, and the recourse to education and educational policies, as instruments to transform this dimension of inequality. To this end, we will seek to identify how the sexual division of labor has been placed as a central vector of social intervention in different programs and initiatives deployed by the organizations for women, especially during the first administration of the National Council for Women. Then, we will try to show the educational responses to the problem of the sexual division of labor developed by the Council in the same period in a context of educational reforms.
\end{abstract}

Keywords: Public policies, gender inequality, work, education.

Fecha de recepción: Febrero 2019

Fecha de aprobación: Diciembre 2019

\title{
Introducción
}

El presente trabajo se enmarca en el proyecto de incentivos denominado "Estado, políticas públicas y desigualdades sexogenéricas. Configuraciones en la Argentina contemporánea (1983-2019)", el cual pretende dar cuenta de las formas de intervención estatal implicadas en la reproducción/ transformación de estas desigualdades y su relación con procesos sociales y culturales más amplios. Uno de los objetivos de este proyecto es el análisis de las políticas de género que se han institucionalizado progresivamente desde el retorno a la democracia en Argentina y América Latina, y las tensiones a las que han dado lugar. Para ello, nos hemos propuesto dar cuenta de cómo algunos aspectos de los marcos interpretativos construidos por los feminismos han logrado permear campos discursivos centrales de las sociedades del capitalismo tardío, como el Estado. Entendemos a estos marcos interpretativos como un "conjunto histórica y culturalmente específico de recursos discursivos de los que disponen los miembros de una colectividad social determinada para plantearse reivindicaciones unos a otros" (Fraser, 2015 [1989]: 79), que se encuentran organizados en sintonía con patrones 
institucionalizados de dominación, y son objeto de lucha cultural y política. En este escrito nos detendremos particularmente en identificar cómo el trabajo es colocado como una dimensión central de la desigualdad de género por los activismos de mujeres y feministas, y cómo se articula esta centralidad con algunas de las propuestas de políticas de género desarrolladas durante los años 90 en Argentina, particularmente las educativas. Nos interesa centralmente identificar cómo algunas de las propuestas analíticas de los marcos interpretativos feministas logran permear los diseños de las políticas, en un contexto donde se combinan paradójicamente una expansión de los derechos formales de las mujeres1 y el desarrollo de políticas de género2, con la consolidación de los regímenes neoliberales en la región.

Partiendo de la concepción de que los feminismos implican tanto la organización y movilización social, como la construcción de teorías para interpretar el mundo, en el primer apartado nos dedicaremos a reconstruir la constitución del marco interpretativo feminista en torno a las desigualdades de género vinculadas al trabajo (Fraser, 2015 [1989]). Para ello, buscaremos mostrar en primer lugar cómo los movimientos de mujeres y feministas de la segunda ola comenzaron a introducir, en una "lengua tartamuda" (Trebisacce, 2016), una terminología novedosa como patriarcado, política sexual y, de particular importancia para nosotres ${ }^{3}$, trabajo doméstico. En segundo lugar, daremos cuenta del pasaje de la lengua tartamuda en que se formularon estas primeras elaboraciones a su incorporación, si bien subordinada, al ámbito de la educación superior y académico, a partir del desarrollo de los estudios de género y feministas.

En el segundo apartado nos dedicaremos a analizar cómo, en un contexto de apertura democrática en América Latina y de expansión del modelo neoliberal a escala global, algunas de las formulaciones feministas logran hacerse lugar en la agenda institucional, apropiándose a la vez que transformando los lenguajes disponibles -de los derechos humanos y las políticas públicas- para enmarcar sus demandas. En este contexto, parte del marco interpretativo propuesto por los feminismos en las agendas activistas y académicas que consideraban al trabajo como un vector de desigualdad, logra permear los organismos internacionales y las políticas públicas en distintos países. Entre ellas, las políticas educativas son ubicadas como centrales para remover los estereotipos

\footnotetext{
${ }^{1}$ La categoría mujer a la que hacemos referencia en el texto tiene ciertamente sesgos cissexista y heteronormativos. Entendemos que este límite es en parte explicado por los propios límites de la categoría mujer y su lugar dentro del feminismo, que fue objeto de fuertes polémicas en los años 80 y 90 . Por eso, antes que sostener una visión esencialista, entendemos que mujer significa cuestiones distintas en diferentes marcos interpretativos operativos en distintos contextos.

2 Usamos de forma laxa expresiones como políticas hacia las mujeres/política de género. Una tipología interesante que rescata matices y complejidades puede encontrarse en Rodríguez Gustá 2008.

${ }^{3}$ Reciente la Facultad de Ciencias Humanas de la Universidad Nacional del Centro de la Provincia de Buenos Aires aprobó el uso de un lenguaje incluyente, no sexista y no discriminatorio. En consonancia con ello, en este trabajo utilizaremos la "e" para evitar el uso del masculino como genérico. Para acceder al documento: http://www.fch.unicen.edu.ar/wpcontent/uploads/2019/11/INFORME-TECNICO-LENGUAJE-INCLUYENTE- 4 .pdf.
} 
que asignan las tareas domésticas y de cuidado a las mujeres, a la vez que bifurcan el mercado laboral en trabajos feminizados y masculinizados.

\section{El trabajo como vector de desigualdad en la agenda teórico-política de los feminismos. Aproximaciones a la construcción de un marco interpretativo}

\subsection{La "lengua tartamuda" del activismo feminista en la denuncia de la opresión de la mujer en el trabajo}

Algunas investigaciones señalan que, desde los años 60 y 70, se producen una serie de transformaciones culturales y de comportamiento que impactaron especialmente en las mujeres, y que plantearon algunas modificaciones en aquellos roles asignados socialmente a mujeres y varones, en un contexto donde el activismo feminista se había ocupado de discutir la idea que unía la sexualidad con la reproducción (Wainerman, 2005). En este sentido y en poco tiempo, se asiste a un aumento de la esperanza de vida, la disminución de la tasa de natalidad, el crecimiento de los divorcios y de hogares monomarentales, a la vez que se observa un aumento del nivel de estudios de las mujeres y su mayor participación en el mercado de trabajo (Arraigada, 1997; Wainerman, 1994).

Las transformaciones sociales y culturales ocurrían en simultáneo con una fuerte radicalización política. La emergencia de las luchas por la liberación en el tercer mundo, de los movimientos negros y el movimiento estudiantil, y la aparición de una nueva izquierda, que ponían en cuestión los cimientos de las sociedades capitalistas, coloniales y racistas occidentales, ofrecieron nuevos espacios de participación política para las mujeres (Arruza, 2015). Sin embargo, como se hizo prontamente evidente, estos espacios fueron resistentes a la incorporación de sus demandas, o a aceptar su organización en espacios propios.

En América Latina, además, este proceso de convulsión social y política coincidía con la instalación progresiva de cruentas dictaduras cívico-militares, que impusieron por la fuerza límites a los procesos de cambio social en curso a nivel global. Incluso, se ensayaba en Chile, en el contexto de autoritarismo de la dictadura pinochetista, los primeros trazos del proyecto neoliberal que se desarrollaría en la región y en el globo en las décadas siguientes (Valdivia, 2010).

La relación entre el feminismo y otros movimientos radicales dependió del contexto. Mientras algunas feministas rompieron con ellos dando lugar a un activismo autónomo, como sucedió con el feminismo radical en Estados Unidos, las reivindicaciones por la igualdad entre varones y mujeres también se hicieron su lugar en organizaciones mixtas a través de un activismo femenino que enfrentó grados variables de resistencia (Nash, 2012; Ergas, 2000). América Latina no fue ajena al dilema de la "doble militancia". Por una parte, surgían grupos autónomos de mujeres en distintos lugares como en 
Argentina (Unión de Feministas Argentina 1970 y el Movimiento de liberación Feminista 1972), México (Movimiento de Liberación de la Mujer 1974 y el Movimiento Feminista Mexicano 1975) (Lau, 2011) y Brasil Associação de Mulheres (1979) (Costa, 2011). Por otra, algunas de las mujeres en las organizaciones políticas y armadas, si bien en minoría y con fuertes limitaciones, comenzaban a impulsar demandas en torno a la "cuestión de la mujer". Esta tensión entre "autónomas" y "políticas" que se delineaba en un contexto de expansión autoritaria, se prolongó luego de la apertura democrática en diversos países, siendo uno de los ejes de tensión de los Encuentros Feministas Latinoamericanos y del Caribe desarrollados desde 1981 (Soporta, Navarro,Chuchryk y Alvarez, 1994).

Como señala Trebisacce (2016), durante la militancia en estas décadas las feministas ensayaron una lengua teórica tartamuda, escribieron libros, panfletos, fanzines donde colocaron temas que estaban invisibilizados en la sociedad en general, articulando un nuevo vocabulario en torno a categorías como patriarcado, supremacía masculina, política sexual y trabajo doméstico, que habilitó también a pensar a las mujeres como el sujeto político de la transformación social.

En este contexto, y en relación con el debate sobre la sexualidad, la anticoncepción y el aborto, el trabajo comienza a ponerse en cuestión como fuente de subalternidad común a las mujeres, especialmente entre feministas o liberacionistas en diálogo con el marxismo. Algunas se centraron en las desiguales condiciones en que mujeres y varones se insertaban en el mercado de trabajo (acceso, segregación horizontal y vertical, brecha salarial), mientras que otras ubicaron el trabajo realizado al interior del hogar (desvalorizado, invisible y mayoritariamente atribuido a las mujeres), como eje fundamental de las desigualdades de género.

Las soluciones propuestas $y$, en algunos casos, ensayadas ante esta problemática, fueron de diverso signo. Organizaciones como la NOW (National Organization of Women) en EEUU bregaron por el acceso de las mujeres al trabajo remunerado y el combate a las desigualdades en este ámbito, realizando por ejemplo asesoramiento legal para litigios con empleadores. Otras reclamaron por una igual distribución de las tareas al interior del hogar entre varones y mujeres, la industrialización del trabajo doméstico y la organización de centros de cuidado infantil autogestionados (Bentson, 1972). Inclusive organizaciones cómo Wages for Housework llegaron a plantear la necesidad de un salario para el trabajo doméstico (Federici, 2013).

\section{Como señalan Theumer y Bellucci:}

"La problematización del trabajo doméstico prolifera política y académicamente a lo largo de los setenta, década comúnmente señalizada como de alta efervescencia social. Ensayistas, académicas y activistas de distintas regiones de Occidente comenzaron a analizar tanto teórica como empíricamente lo que parecía a primera vista un tema superficial: los quehaceres domésticos y el cuidado de la prole. Isabel Larguía y John Dumoulin (1969) en Cuba; Margaret Benston (1969) y Peggy Morton (1970) en Canadá; Christine Delphy (1970) en Francia; Shulamith Firestone (1970) en Estados Unidos; Juliet Mitchell (1966 y 1973) en Inglaterra; María Rosa Dalla Costa y Selma 
James (1972), y después Silvia Federici (1975), en Italia; fueron algunas de las más reconocidas teóricas y analistas que releyeron a Marx y Engels desde el prisma feminista y viceversa. Todas y cada una de ellas tomaron en serio las limitaciones del pensamiento marxiano y buscaron respuestas ante tal vacío teórico." (Theumer y Bellucci, 2018:67).

En América Latina una de las derivas teórico-políticas de esta discusión en torno al trabajo doméstico se encuentra de manera más contundente en el escrito "Hacia una ciencia de la liberación de la mujer" de la argentina Isabel Larguía y el estadounidense John Dumoulin. Les autores realizan una crítica a la producción teórica elaborada por la ciencia económica dominante, en tanto el foco de análisis sobre el funcionamiento del capitalismo se centró en el trabajo productor de mercancías tangibles para ser intercambiadas en un mercado construido en clave masculina. Al observar solo eso, la ciencia económica reproduce en sus elaboraciones teóricas el papel subordinado del trabajo realizado en el hogar, en mayor medida por mujeres. Así, se omitía del análisis el valor económico de este trabajo, así como la consideración a la división del trabajo entre los sexos y sus efectos en la explotación de la mujer. En esta línea Larguía y Dumoulin (1976) van a colocar la mirada analítica en el trabajo doméstico "invisible" y su carácter opresivo. Así, les autores señalan que:

"En el capitalismo, ya sea como propietario de los medios de producción o como operador de los mismos por medio de la venta de su fuerza de trabajo, el hombre se define esencialmente como productor de mercancías. Su posición social se categoriza gracias a esta actividad." (Larguía y Dumoulin, 1976: 13).

En cambio, el trabajo doméstico es invisibilizado, tanto por la sociedad en general como en los esquemas de análisis económico, en tanto el trabajo de reproducción en el hogar aparece como el lugar privado donde se generan bienes de consumo para la satisfacción de necesidades continuas. Así, labores como la limpieza, señalan les autores, crean artículos que carecen de estabilidad, es decir, son efímeros, solo se ven- si es que se ven- momentáneamente y no permanecen en el mundo, a diferencia de los objetos "visibles" creados por el trabajo mercantil de extendido dominio masculino. Sin embargo, pese a esta consideración de invisibilidad del trabajo doméstico, les autores proponen restituir el estatus económico de este trabajo en tanto, señalan, el hogar obrero no se sostiene solo por el aporte del salario masculino. Además de ser un ingreso que apenas garantiza la subsistencia, hay una cantidad de trabajo que el salario no puede hacer. Con él se puede comprar la materia prima para elaborar la comida, pero se requiere de actividades como hacer las compras y cocinar para garantizar la alimentación de les integrantes del grupo familiar. Les autores entienden que con su trabajo cotidiano "las amas de casa reponen diariamente gran parte de la fuerza de trabajo de toda la clase trabajadora (...) El producto invisible del ama de casa es la fuerza de trabajo." (Larguía y Dumoulin 1976: 14 y 16), y esto redunda en enormes beneficios y ahorros tanto para los hombres como centralmente para el capitalismo: "Por lo tanto puede decirse que el 
trabajo femenino en el seno del hogar se expresa transitivamente en la creación de plusvalía, a través de la fuerza de trabajo asalariada" (Larguía y Dumoulin, 1976:14).

Como hemos visto hasta aquí, el MLM colocó tópicos fundamentales en el debate público y la cuestión del trabajo de las mujeres fue uno de los vectores de reclamo y movilización a la vez que se constituyó, como mencionamos antes, en objeto de reflexión teórica. Las discusiones abiertas por el activismo feminista permearon con grados variables de intensidad en las instituciones educativas y centros académicos orientados a la producción de conocimiento sobre la realidad social. Como apunta Trebissace:

“Esta lengua teórica pero artesanal, finalmente tartamuda, interpeló a toda una generación de mujeres, algunas de las cuales ingresaron en los centros universitarios en la década del 80 y comenzaron la tarea de creación de institutos y departamentos que pudieran ser espacios para el desarrollo de estudios críticos con perspectiva feminista" (Trebisacce, 2016: 2).

Según esta autora, se pasaba así de un "lenguaje feminista de la calle" a la disputa "académica y formal” abriéndose una contienda teórica, metodológica y epistemológica, orientada a cuestionar las pretendidas bases neutrales y universales sobre las que descansaba la producción de conocimiento en los grandes centros de investigación.

En el apartado siguiente, nos enfocaremos en algunos de los ejes de ese pasaje de "la calle" a la "universidad" y el ámbito académico de algunos de los planteos feministas. Particularmente, nos detendremos en los cuestionamientos por los que transitó el campo de estudios de la economía observando las modificaciones en torno a la categoría trabajo como resultado del impacto progresivo de los estudios de género y feministas en esta disciplina. Solo vale aclarar que este pasaje no es una instancia definitiva o unidireccional. Los tránsitos y flujos entre activismos y academias han sido una parte constitutiva del despliegue de los feminismos, que ciertamente no han estado (ni están) exentos de conflictos.

\subsection{De la "calle" a la "universidad". Discusiones en torno al trabajo en los estudios feministas/de género y su impacto en la ciencia económica}

Cómo hemos visto, el empuje militante del movimiento de mujeres y feministas de la segunda ola impactó en la forma de entender la familia, la sexualidad, la política y el trabajo, trayendo aparejadas repercusiones que no sólo tuvieron relevancia política, sino que también comenzaron a interpelar los modos académicos de producción de conocimiento. En torno a la cuestión de las mujeres y sus trabajos, en los años 60 y 70 el feminismo instaló un debate que, progresivamente, va a poner en cuestión la visión que había imperado en las ciencias sociales sobre el concepto de trabajo a partir de interrogar la acepción tradicional del mismo (Hirata y Kergoat, 1997) es decir, su equiparación o asociación únicamente con el trabajo asalariado orientado al mercado, poniendo en tela de juicio la división sexual del trabajo en los ámbitos públicos y privados. 
Esta contienda teórico-política abierta por el feminismo llevó a que distintas disciplinas, como la ciencia económica, transitaran por cambios. Los estudios de género y feministas comenzaron a hacerse un lugar en la academia hacia los años '80, logrando permear en los '90 este campo disciplinar cuando aparece la denominada Economía Feminista. Como señala Benería (1999), sus hitos de surgimiento se pueden ubicar en 1992 con la creación de la International Association of Feminist Economics (IAFFE) y en 1995 cuando se publica la revista Feminist Economics (Benerías, 1999).

La Economía Feminista va a retomar problemas vitales como la desigualdad y el trabajo, a partir de reconocer que hay una relación directa entre las relaciones de género y las relaciones económicas y laborales (Rodríguez Enríquez, 2015). Al mismo tiempo va a formular una crítica a la mirada hegemónica de la disciplina económica por sus sesgos androcéntricos, en tanto esta disciplina se centra en las experiencias de los varones en el trabajo asalariado (Carrasco, Galvez y Jubeto, 2016; Larguía y Dumoulin, 1976 [1972]).

Así, los esfuerzos teóricos apuntaron a dejar en evidencia que el trabajo para el mercado se organiza a partir de una división genérica del trabajo (Lipietz, 1996; Hirata y Kergoat, 1997; Rodríguez Enríquez, 2015). La misma refiere a la distribución diferencial del trabajo entre mujeres y varones que se funda en estereotipos de género construidos social y culturalmente y que intervienen tanto al nivel de la oferta, es decir las representaciones de trabajadoras y trabajadores, como del lado de quienes contratan. (Arraigada, 1997; Faur y Zamberlin, 2008)

La diferenciación de tareas y actividades entre mujeres y varones se expresa en tres situaciones. Por un lado, en la segregación ocupacional horizontal (Kessler, 2014) referida a la sobre-representación de las mujeres en algunos sectores como el de servicios (doméstico remunerado, educación y salud), mientras que los varones están concentrados en ciertas actividades como la construcción y el transporte. Por otro lado, la segregación ocupacional vertical refiere a una distribución desigual de mujeres y varones en los puestos de mayor jerarquía en los escalafones ocupacionales, significando que las mujeres están sobre-representadas en puestos de trabajo de "menor calificación" (Todaro; Abramo y Godoy, 2001). Por último, la situación de desventaja de las mujeres se expresaría en las brechas salariales entre aquellas y los varones (Kessler, 2014; Brosio, 2016).

La Economía Feminista también va a revitalizar en el campo de estudios de la economía el debate histórico dentro del feminismo sobre el trabajo doméstico que anticipamos con los aportes de Larguía y Dumoulin (1976). Este aporte apuntó centralmente a poner en discusión el concepto de trabajo que se había empezado a delinear a partir de la industrialización cerrándose en las actividades asalariadas, al visibilizar el rol del trabajo doméstico en la reproducción social (Federici, 2013). Si bien el aumento progresivo de las mujeres en el "mercado de trabajo" corría las fronteras de los ámbitos público-masculino y doméstico-femenino, al interior del hogar, los roles y actividades generizadas no experimentaban los mismos corrimientos (Hoschschild, 2008). El trabajo doméstico seguía apareciendo como un trabajo de mujeres (Federici, 2013). Con lo cual, en muchos casos, estas optaban por trabajos flexibles, más precarios, para conciliar el trabajo asalariado y la familia resignando tiempo y actividades para sí mismas 
(Wainerman, 2005; Hoschschild, 2008). Esto genera una sobrecarga de trabajo tanto a nivel físico como psicológico (Anzorena, 2013a), que redunda en la sobreexplotación de las mujeres, en un fenómeno conocido como "segunda" o "doble jornada" (Wainerman, 2005; Hoschschild, 2008).

Esto dio lugar también, en las últimas décadas, a la emergencia del cuidado como objeto de estudio (Esquivel, 2011), que refiere a las actividades necesarias para la reproducción cotidiana de las personas tales como el cuidado de personas dependientes y las tareas del hogar necesarias para cuidar como la limpieza, los mandados, cocinar (Rodríguez Enríquez, 2015). Este trabajo invisible, como destacaron Larguía y Dumoulín (1976) es fundamental en tanto "la economía del cuidado sostiene el entramado de la vida social humana (...) y se constituye en la base del edificio económico" (Bosch en Carrasco, 2014a: 46). Para la Economía Feminista el trabajo doméstico y de cuidados es uno de los núcleos centrales que sostiene la división del trabajo por género. Su distribución asimétrica, entre varones y mujeres, pero también entre instituciones como los hogares, el Estado, mercado y organizaciones comunitarias representados en la figura del "diamante del cuidado", se considera una traba en la participación económica de las mujeres, lo que refuerza desigualdades de clase y género (Rodríguez Enríquez y Pautassi, 2014). Por ello, era central visibilizar política y teóricamente su papel económico y social, a la vez que esto buscaba incidir en la desnaturalización de estas tareas como actividades "de mujer".

Como veremos en los próximos apartados, particularmente para el caso de Argentina podemos rastrear que, de la mano del decenio de la mujer y de las recomendaciones internacionales sobre los derechos de las mujeres, algunas de las discusiones teóricas referidas a la posición económica subordinada de las mujeres y a la ampliación del concepto de trabajo permearon los procesos de institucionalización de las políticas "para la mujer" o "de género". Especialmente después de la Conferencia Internacional de la Mujer que se realizó en Nairobi, la necesidad de desarrollar organismos específicos que abordaran la cuestión de la mujer se planteó como prioritaria. Si de la mano de su incursión en el ámbito académico y la educación superior, los debates feministas lograron construir un marco interpretativo para problematizar el carácter generizado del trabajo, en estos organismos la educación apareció como uno de los medios posibles para erosionar las desigualdades de género.

\section{De la producción teórica a la política pública.}

\subsection{Entre lo global y lo local. La institucionalización de las políticas de género}

Los años 80 marcan el fin de los regímenes dictatoriales que se impusieron en el cono sur siguiendo el programa militar que instaló el general Pinochet en Chile tras derrocar, por medio de un golpe de Estado en 1973, al gobierno de la coalición de izquierda Unidad Popular, encabezado por Salvador Allende (Valdivia, 2010; Tinsman, 2016). El proceso de apertura democrática que se inicia en América Latina es 
acompañado por una extensa movilización política de distintos sectores sociales y un fuerte cuestionamiento a los crímenes desatados por las dictaduras de la región. En este contexto de elevada politización social, se comienzan a desarrollar los Encuentros Feministas Latinoamericanos y del Caribe, dando lugar a importantes instancias de intercambios y discusiones entre feministas latinoamericanas, que continúan hasta la actualidad. En los primeros cinco encuentros desarrollados en Bogotá (1981); Lima (1983); Bertioga (1985); Taxco (1997) y San Bernardo (1990) se puede observar cómo, en los talleres y propuestas de actividades, se retomaron ejes de discusión específicos en torno a la sexualidad, el respeto por los derechos humanos, la situación de la mujer en el trabajo asalariado y doméstico; educación, Estado y políticas públicas en torno a las mujeres (Saporta; Navarro; Chuchryk y Álvarez, 1994). Con matices según la zona y los contextos, algunos de estos tópicos ya estaban presentes en la agenda que abrieron los feminismos de la segunda ola, tanto en la región como en Estados Unidos y diversas partes de Europa.

Un debate entre las feministas latinoamericanas, que adquiere un nuevo signo por el cambio del contexto dictatorial, es el referido a la oposición entre desarrollar una política feminista autónoma, o intervenir como feministas no sólo en organizaciones mixtas, sino también en espacios abiertos por los procesos de democratización como la expansión de las ONG, la creación de dependencias estatales específicas, y la participación en organismos internacionales. Si en la década del 60 y 70 se fortalecía la posición de renuncia a un diálogo con diversas instancias institucionales, el nuevo escenario habilitó a que algunas organizaciones feministas empezaran a ver con otros ojos su relación con el Estado (Saporta; Navarro; Chuchryk y Álvarez, 1994; Lau, 2011). Por otra parte, en este contexto, el lenguaje de los derechos humanos y las políticas públicas se volvió una lengua franca para movimientos sociales con diversas agendas e intereses.

Los movimientos y organizaciones de mujeres y feminista, en algunas de sus variantes, encontraron en la apertura de espacios institucionales y el lenguaje de los derechos humanos el acceso a un conjunto de recursos discursivos para plantear sus demandas, a la vez que intentaron permear sus propios marcos interpretativos en estas instancias (Fraser, 2015 [1989]). En este contexto, la emergencia de una experticia feminista tanto en espacios de gestión como académicos se convirtió en puente (Fraser, 2015 [1989]) entre las reivindicaciones y demandas impulsadas en espacios como en los Encuentros Feministas Latinoamericanos y del Caribe y los Encuentro Nacionales de Mujeres de Argentina y el desarrollo de políticas públicas de género.

Para el caso del derrotero de institucionalización progresiva de los derechos de las mujeres, es común marcar como un punto de inflexión importante algunas Conferencias Mundiales desarrolladas por Naciones Unidas entre 1974 y 1995. Nos referimos particularmente a las Conferencias Mundiales sobre la Mujer desarrolladas durante el decenio de la mujer entre 1975 y 1985, las Conferencias sobre Población y Desarrollo de Bucarest (1974) y El Cairo (1994), y la Conferencia Mundial sobre Derechos Humanos de Viena (1993). En estos espacios, por primera vez, se reconocían los derechos de las mujeres como derechos humanos (Facio, 2011). Las demandas que habían transitado del 
ámbito invisible de lo doméstico, al escenario visible de la vida pública a partir de procesos de politización, fueron progresivamente incorporadas en la agenda institucional (Fraser, 2015 [1989]). Se incluyeron cuestiones como la salud sexual y reproductiva de las mujeres como alternativa a las políticas de población anti o pronatalistas, la participación política y social en condiciones de igualdad, el acceso a la educación y la modificación de los patrones de género que esta contribuye a reproducir, y la participación económica de las mujeres, tanto en el trabajo asalariado como en el hogar.

La aprobación en 1979 de la Convención sobre la eliminación de todas las formas de discriminación contra la mujer (CEDAW) constituyó una condensación normativa de estas demandas, que resultaría de fundamental importancia para el desarrollo de políticas de género. Esta normativa define a la discriminación en su preámbulo como "toda distinción, exclusión o restricción basada en el sexo (...) en las esferas política, económica, social, cultural y civil o en cualquier otra esfera" (CEDAW, 1979: 10). En función de los intereses este artículo es central señalar cómo esa definición de discriminación se especifica en lo relativo al ámbito del trabajo. En el artículo 11, se promueven "medidas apropiadas para eliminar la discriminación contra la mujer en la esfera del empleo" por razones de "matrimonio o maternidad" y se llama a los estados a incorporar la licencia por maternidad paga y promover servicios sociales necesarios para conciliar responsabilidades familiares, obligaciones del trabajo y la participación en la vida pública (CEDAW, 1979: 15). En la Convención, la discriminación en el ámbito del trabajo se vincula directamente con la discriminación en el ámbito educativo. Tal como se explicita en el artículo 10, en donde, entre otras cosas, se coloca la necesidad de garantizar "Las mismas condiciones de orientación en materia de carreras y capacitación profesional, acceso a los estudios y obtención de diplomas" y eliminar las representaciones estereotipadas de los roles masculinos y femeninos" (CEDAW, 1979: 13)

A la condensación normativa que supuso la CEDAW, se le sumaron los impulsos por desarrollar dependencias estatales específicas. A partir de la Conferencia que clausuró el decenio de la mujer, realizada en Nairobi, Kenya en 1985 y sus "Estrategias de Nairobi para el Adelanto de las Mujeres", se dio un viraje respecto de las orientaciones anteriores, que se profundizará con las conferencias subsiguientes. Según Anzorena:

"Los gobiernos, a través de la adhesión a este documento, se comprometían a implementar medidas que fomentaran la participación de las mujeres en la promoción de la paz, en la asistencia a mujeres en situación de riesgo y en una amplia gama de temas: empleo, salud, educación y servicios sociales, industria, ciencia, comunicaciones y medio ambiente" (ONU, 2008 en Anzorena, 2013 b: 62).

Entre esas medidas se recomendaba el establecimiento de mecanismos gubernamentales apropiados para el seguimiento y la mejora de "la situación de la mujer".

"También se puso énfasis en que para ser efectivos debían estar establecidos en un alto nivel de gobierno y garantizarse los recursos, el compromiso y las autoridades 
adecuadas para asesorar sobre el impacto "en las mujeres" de todas las políticas gubernamentales" (ONU, 1986, par. 57en Anzorena, 2013b: 62).

En Argentina estas discusiones se dieron en un contexto de apertura democrática y de profundas transformaciones políticas, económicas y culturales. Las décadas de los '80 y '90 estuvieron atravesadas por la discusión en torno al deterioro progresivo de las condiciones de vida, la transformación del Estado y el lugar que debía ocupar la educación en este contexto. Pero también fueron el escenario de revitalización de un movimiento de mujeres y feminista, que tuvo como dos de sus marcas de época los Encuentros Nacionales de Mujeres y la institucionalización de algunas de demandas de género en marcos legislativos y organismos específicos (Barrancos, 2007).

El marco habilitado por la expansión del lenguaje de los derechos humanos se encarnó en algunas transformaciones normativas que tuvieron lugar entre mediados de los 80 y los 90 . En este lapso se avanzó en el terreno legislativo con la aprobación de leyes como el divorcio vincular (ley 23515/87), y la protección contra la violencia familiar (ley 24417/95). Por otra parte, la sanción de la ley 23179 que ratificaba a la Convención contra Todas las Formas de Discriminación contra la Mujer (CEDAW) aprobada por la ONU en 1979, apuntaló el proceso de desarrollo de políticas de igualdad de oportunidades en clave de acción positiva, cuya expresión más característica fue la ley de cupo femenino aprobada en 1991. Y en 1994 el proceso de reforma constitucional habilitó un escenario de posibilidades de reconocimiento jurídico, aunque atravesado por duros conflictos. En su conjunto, estos avances muestran cierta erosión de un orden de género tradicional, posible por la expansión y utilización del lenguaje de los derechos (Pecheny, 2014).

Las propuestas establecidas en Nairobi y los recientemente creados Encuentros Nacionales de Mujeres, cuya primera edición fue en 1986, tuvieron su impacto en el diseño de organismos y burocracias específicamente creadas para transformar las relaciones de género. En 1987 el programa Promoción de la Mujer y la Familia, que funcionó en el ámbito del Ministerio de Salud y Acción Social, fue sucedido por la Subsecretaría de la Mujer, que contó con tres direcciones nacionales, y una planta de algo más de cuarenta personas. La subsecretaría busco alejarse de las políticas de corte exclusivamente asistencial asociado a las mujeres, desarrollando líneas de acción en consonancia con las que se marcaban a nivel internacional y con algunos de los ejes que planteó el feminismo argentino en décadas pasadas: educación, salud, trabajo, vida cotidiana y familia, legislación y medios de comunicación. Estratégicamente, la línea principal buscó dar visibilidad a las problemáticas de las mujeres en distintos ámbitos, realizando capacitaciones y líneas de investigación (Barrancos, 2007). A cargo de esta dependencia estatal estuvo Zita Montes de Oca, una figura clave del movimiento feminista y de mujeres desarrollado con posterioridad al retorno democrático, especialmente con mujeres de diversos partidos políticos (Thiteus Atschil, 2015).

Con la asunción de Carlos Menem como presidente de la nación en 1989, la Subsecretaría fue cerrada para crearse en 1990 el Consejo Coordinador de Políticas Públicas para la Mujer (CNM), mediante el decreto 378, y luego en 1992, por el decreto 
1426, el Consejo Nacional de la Mujer dependiente directamente del poder ejecutivo. Su objetivo principal sería dar cumplimiento a la CEDAW (artículo 2) y tomar intervención en todos los proyectos y acciones vinculados con la cuestión de la mujer (artículo 18). Se designó como presidenta a Virginia Franganillo, una socióloga egresada de la Universidad de Mar del Plata, militante peronista y activa en el movimiento de mujeres a fines de los ochenta y a comienzos de los noventa, particularmente en la Multisectorial de la Mujer 4 .

Según Franganilllo ${ }^{5}$, el Consejo Nacional de la Mujer (CNM) recogió la experiencia de la Multisectorial, de los Encuentros Nacionales de Mujeres, que se realizaban desde 1986, y de propuestas similares de políticas públicas, como el Consejo Provincial de la Mujer de la provincia de Buenos Aires, creado en 1987 e inspirado en el modelo ideado por feministas brasileras.

Ahora bien, este derrotero de institucionalización de los derechos de la mujer en organismos y políticas estatales acontece en un escenario paradójico. Cómo advierte Brown:

"La pregunta que reaparece es qué democracia es posible en un contexto en el que se produce el efecto paradójico de una ampliación de derechos formales para colectivos otrora excluidos, como las mujeres, a la vez que se deterioran las condiciones de vida para amplias mayorías y el Estado se retrae cada vez más dejando en conjunto progresivamente mayor de bienes y derechos librados al mercado" (Brown, 2014: 77).

Para el caso de Argentina, según la concepción neoliberal que se venía imponiendo desde la última dictadura militar, el Estado debía ser reducido a sus funciones esenciales (seguridad, justicia, defensa, relaciones exteriores y administración), es decir que debía achicarse y racionalizarse. Así, entre las décadas del ' 80 y ' 90 , los procesos de ajuste estructural y flexibilización laboral que se llevaron adelante se expresaron en el crecimiento de los niveles de desempleo y la caída del salario real, aumento de la inflación a la vez que crecieron los niveles de desempleo, informalidad y la pobreza, deteriorando las condiciones de vida y el bienestar de vastos sectores de la población. En este contexto de "crisis del trabajo" las mujeres asumen de manera creciente un espacio mayor en el mercado de trabajo (Wainerman, 2005) especialmente entre los sectores de menores ingresos (Cutuli, 2012), a la par que aquel se precariza e informaliza. Estos temas emergen con fuerza cuando los varones, con más trayectoria en el trabajo asalariado formal, comienzan a experimentar el desempleo y la precarización de sus relaciones laborales, situación que, si bien no era novedosa para las mujeres y otros grupos sociales (Carrasco, 1997), se complejiza en el nuevo contexto neoliberal.

En este apartado nos hemos referido a los procesos que, entre lo global y lo local, colocaron en primer plano algunas de las demandas de las mujeres y feministas, algunas

\footnotetext{
4 Fue creada en Capital Federal, Argentina luego del 8 de marzo de 1984. Estaba integrada por mujeres de diversa procedencia social, sindical y política.

${ }^{5} \mathrm{Al}$ igual que había sucedido con el acceso al voto para las mujeres, la creación por decreto del Consejo preocupó a varias feministas. Aunque se realizaron intentos, ratificar su creación mediante una ley, estos no prosperaron (Barrancos, 2007).
} 
de las cuales recurrieron al lenguaje de los derechos y las políticas públicas en franca expansión a nivel global, y de mucha pregnancia en un contexto regional posdictatorial. De la mano de activistas y expertas feministas que participaron de espacios institucionales, se tendió un puente y una traducción entre las demandas de algunos grupos sociales y el desarrollo de políticas públicas que buscaron erosionar un orden de género tradicional y desigual. En este marco deben entenderse las transformaciones normativas y la creación de organismos de/para la mujer en la Argentina de los '80 y '90. Sin embargo, y como mencionamos antes, estas conquistas en clave institucional ocurren paradójicamente en un contexto donde el empleo, que había sido vehículo de acceso a derechos y bienestar económico, en principio para los varones, ya no cumplía con esas funciones, que había asumido en el contexto de excepción histórica de la sociedad salarial (Méda, 1998). Si bien para las mujeres el desempleo y la precarización de sus relaciones laborales eran características más extendidas, el contexto de deterioro general de las condiciones económicas y de trabajo impactó con mayor intensidad sobre todo entre las de menores recursos.

A continuación analizaremos uno de los programas desarrollados por el Consejo Nacional de la Mujer, destinado al área educativa. Intentaremos mostrar cómo algunas de las herramientas interpretativas propuestas por el feminismo y los estudios de género respecto de la organización económica y el trabajo, lograron salir de los claustros académicos o de sus formulaciones exclusivamente teóricas en el marco de las universidades, para intentar permear las propuestas de reforma educativa. Buscaremos poner en evidencia cómo algunos de los intentos por transformar los patrones culturales transmitidos en el ámbito educativo, se preocuparon particularmente por la relación entre género y trabajo. Todo esto en un contexto paradójico de ampliación de derechos y expansión de políticas neoliberalismo.

\subsection{Género y trabajo en el Programa de Igualdad de Oportunidades para la Mujer en el Área Educativa (PRIOM)}

El impulso de las perspectivas feministas también logró permear, al menos en parte, las discusiones teóricas y políticas vinculadas a la educación. La preocupación respecto de cómo la escuela participa de la reproducción y transformación de las relaciones de género, comenzó a adquirir peso en este momento. Entre las cuestiones que comenzaban a ser problematizadas se encontraban la invisibilización del trabajo doméstico como trabajo y su vinculación exclusiva con lo femenino, la socialización diferencial de varones y mujeres, y los estereotipos respecto de trabajos femeninos y masculinos. Esto podía evidenciarse tanto en el currículum explícito, manuales y libros de texto como en la práctica cotidiana en las escuelas (Wainerman y Barck de Raijman, 1987; Wainerman y Heredia, 1999; Morgade, 2001).

El reconocimiento de esta participación de la educación en modelar de forma generizada los contornos de la organización económica y del trabajo, llevaron a que el CNM desarrollara una política específica para esta área. Mediante con el Ministerio de Educación y el Instituto Nacional de la Administración Pública, se creó el Programa de 
Igualdad De Oportunidades para la Mujer en el Área Educativa (PRIOM). Este programa fue coordinado por Gloria Bonder y Graciela Morgade, ambas figuras centrales en el desarrollo de la perspectiva de género en educación en Argentina y América Latina. Según Graciela Morgade, quien fue su coordinadora operativa, el PRIOM estaba inspirado en las demandas del movimiento de mujeres y feminista, y sustentado en una visión que entendía al Estado tanto en su faceta patriarcal y reproductora de desigualdades de género, como una plataforma necesaria para reclamar igualdad y un espacio que ofrece posibilidades democratizadoras (Morgade, 1998).

En el Documento de Trabajo $N^{\circ} 1$ de 1992 el Programa reconoció la tendencia a la relativa paridad en el acceso al sistema educativo para varones y mujeres, advirtiendo al mismo tiempo que:

"Sin embargo, la desigualdad es un fenómeno constatado en la práctica y se manifiesta fundamentalmente en cómo se educa a niños-as y jóvenes. Es decir, en los mensajes y valores que se les transmiten acerca de su futuro papel en la sociedad a través de los contenidos curriculares, los libros escolares, las actitudes de los docentes, etc. Con frecuencia, en éstos se adscriben a varones y mujeres determinadas características psicológicas y formas de comportamiento y se les asignan roles sociales fijos por el solo hecho de pertenecer a un determinado sexo. Como es evidente, de esta manera estamos restringiendo y condicionando la posibilidad de desarrollo pleno de todos los sujetos, sean estos varones o mujeres." (PRIOM, 1992:4)

Más adelante en el mismo texto, se menciona esta distinción haciendo referencia al sexo como una condición biológica que distingue entre macho y hembra, diferenciado del género, al que se alude como las características sociales y psicológicas atribuidas al sexo, que jerarquizarían al polo "masculino" frente al "femenino". Bajo esta definición de desigualdad, el programa se propuso como objetivos tanto la incorporación a la reforma educativa de enfoques que promuevan la equidad de género, como resaltar los aportes desarrollados por las mujeres en distintos ámbitos. Para ello se realizaron programas de sensibilización y capacitación, implementación de proyectos de investigación, elaboración y difusión de material educativo, la inclusión de la igualdad de género en programas de formación docente, textos y programas (PRIOM, 1992:8).

Siguiendo los objetivos anteriormente mencionados el PRIOM se planteó la revisión de las formas de socialización de género presentes en la escuela haciendo foco en la organización y los materiales escolares (libros de texto, manuales) y la definición de los Contenidos Básicos Comunes. Las desigualdades de género vinculadas al trabajo fueron una de las aristas sobre las que el programa se propuso intervenir. En sintonía con algunos de los desarrollos de la teoría feminista que recuperamos en el apartado anterior, el PRIOM se orientó a transformar las representaciones culturales estereotipadas, que ubicaban a las mujeres principalmente en trabajos y profesiones "femeninas", y les asignaban en mayor medida el trabajo doméstico y de cuidados.

En lo que sigue buscaremos identificar, a partir de propuestas de capacitación docente y de lineamientos curriculares elaborados en el marco del programa, los intentos 
por trasversalizar la perspectiva de género en el área educativa. Analizaremos más específicamente, algunas de las formas concretas en que el PRIOM se propuso transformar diversas aristas de la división sexual del trabajo, que se constituyeron en ejes de desigualdad entre varones y mujeres.

Como señalamos al finalizar el apartado anterior, nos proponemos recuperar algunas iniciativas desarrolladas por el PRIOM para incorporar la perspectiva de género en educación. Dado el objetivo de este trabajo, nos enfocaremos particularmente en cómo el programa se propuso alterar las desigualdades articuladas en la relación génerotrabajo. Lo haremos recuperando, en un primer momento, dos propuestas orientadas a la formación docente, y en el segundo, un documento diseñado para la incorporación de la perspectiva de género a los Contenidos Básicos Curriculares ${ }^{6}$.

\subsubsection{Perspectiva de género en la formación docente}

Para el año 1993 la coordinación del programa elaboró dos manuales denominados "La igualdad de oportunidades para varones y mujeres en el sistema educativo: una meta educativa" y "Educando a mujeres y varones para el S.XXI". Ambas propuestas buscaron constituirse en un aporte teórico- práctico orientado a intervenir en la formación de docentes y planificadoras de los institutos de capacitación docente, a las cuales se les reconoce su papel como agentes activos en la construcción de relaciones más equitativas entre los géneros y de prácticas educativas orientadas a la igualdad de oportunidades entre varones y mujeres.

Como hemos visto, un ámbito en el que se expresa la posición subordinada de las mujeres es el mundo del trabajo, y por ello, entre los vectores conceptuales básicos sobre los que descansan los manuales, se retoman algunos de los aportes teóricos del feminismo académico que colocamos en el segundo apartado. En este sentido, en ambos manuales de formación se señala la centralidad que tiene la identificación de los estereotipos de género predominantes en nuestras sociedades y que se transmiten por medio de la educación, y su incidencia en las elecciones de estudio y trabajo de mujeres y varones. Por ello, algunos de los contenidos que se proponen para trabajar, refieren a la orientación vocacional y profesional, representaciones y mitos sobre el mundo del trabajo y la futura inserción laboral de varones y mujeres; obstáculos y conflictos de las mujeres

\footnotetext{
6 A partir de la sanción de la Ley Federal de Educación en 1993, la estructura del sistema educativo quedaba conformada por la Educación Inicial, una Educación General Básica obligatoria de nueve años, que dividida en tres ciclos de tres años que abarcaron de primer a noveno año, y una Educación Polimodal de tres años de duración como mínimo, la Educación Superior, profesional y académica de grado y la Educación de Posgrado. Según consta en su segunda edición, los Contenidos Básicos Curriculares "(...) forman parte de los acuerdos federales para la Transformación Curricular y constituyen la definición del conjunto de saberes relevantes que integrarán el proceso de enseñanza de todo el país. Los CBC son la matriz básica para un proyecto cultural nacional; matriz a partir de la cual, cada jurisdicción del Sistema Educativo continuará actualizando sus propios lineamientos o diseños curriculares y dará paso, a su vez, a diversos pero compatibles proyectos curriculares institucionales" (CBC, 1995:17).
} 
en el ejercicio de la maternidad y en el trabajo doméstico, concepciones sobre la participación económica, política y social de mujeres y varones, etc.

Si bien los manuales proveen herramientas para abordar distintos ámbitos en los que la desigualdad de género se expresa, en lo que sigue nos enfocaremos en las propuestas metodológicas de ambos. En estas se proponen algunos tópicos de reflexión en torno al trabajo de mujeres y varones, a la vez que se describen algunas actividades y recursos orientados a desandar roles y estereotipos rígidos de género vinculados al ámbito del trabajo.

De esta manera, ambas propuestas contemplan una guía de actividades orientada a identificar los estereotipos en los materiales y textos de lectura escolar. Para ello proponen por ejemplo que, en grupo, las y los docentes analicen los textos que usan en el grado al que corresponde cada una aplicando una guía de análisis y entre las preguntas figuran: ¿cuántos varones y mujeres aparecen?, ¿qué actividades desempeñan unos y otras?, ¿juegan ellas papeles importantes?; ¿se las ve en ocupaciones tradicionalmente masculinas? ¿y en puestos de decisión?, ¿aparecen varones desempeñando tareas tradicionalmente femeninas?; ¿quienes realizan el trabajo doméstico y se ocupan de la crianza de las y los niños?, cuando los varones realizan tareas domésticas ¿se los muestra positivamente o se los ridiculiza?, ¿aparecen mujeres destacadas en la literatura, ciencia y filosofía?; ¿se recomiendan libros escritos por mujeres en la bibliografía? Por último, se propone también seleccionar alguna lectura estereotipada y redactar una alternativa no discriminatoria.

Como hemos visto más arriba, uno de los aportes de las feministas académicas respecto de estos temas tuvo que ver con mostrar cómo las prefiguraciones socioculturales en torno al género y al trabajo operan haciendo aparecer a mujeres y varones en ámbitos de trabajo valorados social y económicamente de forma diferencial. En este sentido, hemos identificado tres ejes en los que las desigualdades entre los géneros en el mundo del trabajo, entendido en términos amplios, son abordadas en las propuestas.

Por un lado, en ambos manuales encontramos actividades orientadas a trabajar estereotipos en las expectativas y prácticas escolares vinculados a la inserción ocupacional diferencial de mujeres y varones en el mercado de trabajo. Así, en la propuesta Educando a varones y mujeres en el S. XXI se propone a las y los docentes como actividad imaginar la tarea de repartir 10 becas para un taller de computación, 10 para uno literario y otras 10 para un taller de plomería. Se les pide que coloquen los nombres de las y los alumnos seleccionados para cada taller con el objetivo de analizar los prejuicios y estereotipos o las innovaciones presentes en la selección de las y los docentes y reflexionar sobre sus motivos. En esta misma línea otra actividad apuntó a que docentes escribieran adjetivos que se asocian a "los varones son..." y las "mujeres son" para discutir si se trata de características aprendidas o naturales, y luego de la puesta en común jugar reemplazando el "son" por el "educamos a varones y mujeres para". Por otra parte, en el manual "La igualdad de oportunidades para mujeres y varones" aparecen preocupaciones similares a las planteadas anteriormente. Entre los tópicos para la reflexión docente que propone se encuentra, por ejemplo, la participación de varones y 
mujeres en trabajos estereotipados y de distinta jerarquía social (varones médicos, pilotos de avión, gerentes de banco y mujeres enfermeras, azafatas, secretarias).

Otro foco de preocupaciones está en el aporte social y económico del trabajo doméstico y de cuidados realizado al interior del hogar en mayor medida por las mujeres y las asimetrías que reproduce su desigual distribución entre mujeres y varones. El manual antes mencionado coloca algunas preguntas para reflexionar, por ejemplo, si varones y mujeres participan en la misma medida del trabajo doméstico, o si padres y madres asisten por igual a las reuniones escolares. Al mismo tiempo, en los programas y contenidos para las ciencias sociales aparecen interrogantes vinculados a los estudios sobre el trabajo, como por ejemplo si se tiene en cuenta el trabajo doméstico y su importancia para la economía del país.

Por último, otra de las cuestiones que se proponen trabajar ambos manuales refieren a la reflexión en torno a la actividad docente como un trabajo generizado. Así, el manual Educando a mujeres y varones para el S.XXI para ver cómo inciden las construcciones estereotipadas sobre el trabajo de mujeres y varones en las elecciones profesionales de Ixs docentes, una de las propuestas residió en hacerles escribir en una hoja de forma individual y anónima respuestas a: ¿porque soy maestro/a?; ¿quise seguir otra carrera? ¿cuál? ¿por qué no la elegí o no la ejerzo? ¿qué estudiaron los varones de mi familia? ¿y las mujeres?; ¿cómo me imagino en lo profesional en unos años? Por otra parte el manual "La igualdad de oportunidades entre mujeres y varones" propone a los docentes reflexionar en torno a la docencia como un trabajo en el que predominan las mujeres, pero cuyos cargos directivos y de conducción gremial y altos cargos de conducción y planificación de la política educativa están casi exclusivamente en manos de varones.

Como puede verse, la formación docente fue uno de los vectores estratégicos a partir de los cuales el PRIOM se propuso incidir en los procesos de socialización escolar de género, en un contexto de institucionalización de los derechos de las mujeres. Formando a les docentes a partir de los aportes de la perspectiva de género, se esperaba poder transformar la sociedad en general, trastocando los roles tradicionales asignados a varones y mujeres. En particular, como hemos visto, en las actividades y contenidos propuestos en los manuales de formación, subyacían algunos de los aportes de los marcos interpretativos a través de los cuáles los feminismos y los estudios de género reformularon el análisis de la organización económica y el trabajo. En este sentido, a partir de estas propuestas, se buscó dotar a les docentes de un repertorio de recursos discursivos novedosos, a partir de los cuales interpretar y transformar las realidades sociales, dentro y fuera de la escuela.

Ahora bien, la formación docente no era el único ámbito en el que resultaba estratégico intervenir. El proceso de reforma educativa que se desarrolló en Argentina en los años 90, constituyó una oportunidad inusitada para que las expertas encargadas del programa, intentaran incidir de forma más general en el rumbo que tomaría la política educativa. La discusión en torno a los Contenidos Básicos Curriculares para la Educación General Básica fue un escenario donde se condensaron los intentos por trasladar los marcos interpretativos feministas para comprender la socialización de género, en 
particular la relación entre género y trabajo, e intentar transformarla. A este proceso dedicaremos el siguiente momento del trabajo.

\subsubsection{El género en los Contenidos Básicos Curriculares}

Otra instancia que se constituyó en una oportunidad para los intentos del PRIOM de transformar las representaciones estereotipadas de varones y mujeres en el mundo del trabajo, fue el proceso de discusión sobre los Contenidos Básicos Curriculares que coordinó el Consejo Federal de Educación en 19937. En sus resoluciones 26 y 33, donde se aprobaron las orientaciones generales, propuesta metodológica y orientaciones básicas para acordar los Contenidos Básicos Comunes, se planteó que estos debían ser producto de una deliberación democrática, que incluyera la participación de expertes. En este marco, el PRIOM elaboró una propuesta para incorporar de forma transversal la perspectiva de género en los Contenidos Curriculares Básicos. Respecto de la participación del programa en el proceso de transformación curricular, su directora Gloría Bonder mencionaba:

"En este momento, y en el marco del proceso de descentralización y del nuevo modelo educativo que se está impulsando en la Argentina, se están revisando la currícula, entonces, nuestro aporte es participar en las reuniones en que esto se está llevando a cabo" (Bonder, 1993:123).

En este marco, Gloria Bonder y Graciela Morgade elaboraron un documento de cuarenta y dos páginas denominado Propuestas para integrar los aportes de los Estudios de la Mujer a los Contenidos Básicos Curriculares como un aporte para la determinación de los CBC. En él se desarrollaba la propuesta de transversalización de la perspectiva de género elaborada en el marco del programa. En el primer apartado "La perspectiva del campo disciplinario de los Estudios de la Mujer", se sintetizaba el marco interpretativo que promovía el programa, definiéndose el concepto de género y su implicancia en los procesos sociales, particularmente en la adquisición de identidades y roles en la familia, la escuela y el trabajo.

Entre algunos de los puntos de cuestionamiento, y de las propuestas de innovación curricular, encontramos intentos de modificar los sentidos en torno al trabajo y

\footnotetext{
${ }^{7}$ A partir de la sanción de la Ley Federal de Educación en 1993, la estructura del sistema educativo quedaba conformada por la Educación Inicial, una Educación General Básica obligatoria de nueve años, que dividida en tres ciclos de tres años que abarcaron de primer a noveno año, y una Educación Polimodal de tres años de duración como mínimo, la Educación Superior, profesional y académica de grado y la Educación de Posgrado. Según consta en su segunda edición, los Contenidos Básicos Curriculares "(...) forman parte de los acuerdos federales para la Transformación Curricular y constituyen la definición del conjunto de saberes relevantes que integrarán el proceso de enseñanza de todo el país. Los CBC son la matriz básica para un proyecto cultural nacional; matriz a partir de la cual, cada jurisdicción del Sistema Educativo continuará actualizando sus propios lineamientos o diseños curriculares y dará paso, a su vez, a diversos pero compatibles proyectos curriculares institucionales" (CBC, 1995:17).
} 
el género. Por ejemplo, se señalaba respecto de los manuales escolares y demás recursos educativos que estaban permeados por estereotipos de género con una diversidad de expresiones, haciendo referencia a las cualidades físicas, intelectuales, valorativas, afectivas o a la situación social de varones y mujeres, que tendían a reforzar el lugar subordinado de las últimas. Las mujeres, señalaron estas autoras, siempre aparecieron representadas como esposas o amas de casa, reservando a los varones el papel protagónico en las lecturas. Los padres son representados como personajes poderosos, mientras que las madres son débiles y emotivas. Como vimos más arriba, la reflexión sobre cómo se retoma la diferencia sexual para la organización de un mercado de trabajo desigual en términos de reconocimiento social y económico entre los trabajos "femeninos" y "masculinos" fue central para los estudios de género y feministas. En esta línea, las autoras encuentran que en los manuales escolares las actividades laborales suelen restringirse al ámbito doméstico no remunerado, y en las ocasiones en que las mujeres son representadas en actividades remuneradas, se las vincula a "trabajos femeninos". Los manuales escolares también revelaban estereotipos discriminatorios, tal era el caso, por ejemplo, de la historia, que excluía la vida cotidiana e invisibilizaba la participación de las mujeres en movimientos sociales y la producción de cultura.

Este último punto se conectó con la preocupación por la orientación vocacional y profesional. Respecto de la misma se señaló que, aunque no se contaba con una evaluación de su impacto, solía considerarse que las representaciones sobre el mundo del trabajo presente en la escuela constituían elementos de peso en la elección de opciones laborales. Por ejemplo, algunas investigaciones recuperadas en este documento daban cuenta que, con frecuencia, de forma directa o indirecta, los varones eran orientados hacia las ciencias naturales y las mujeres hacia las humanidades. Por último, también se hacía alusión al carácter generizado de la organización y las estructuras escolares en donde, pese a haber alcanzado la paridad entre varones y mujeres en el acceso a todos los niveles del sistema educativo, ésta no se constataba en cargos jerárquicos, ocupados principalmente por varones.

En la segunda parte del documento ${ }^{8}$, "Contenidos propuestos", se desarrolla la propuesta de incorporación del enfoque de género e igualdad de oportunidades de

\footnotetext{
${ }^{8}$ Antes de desarrollar los contenidos en función de las áreas y niveles, en el documento se presenta una gradación de la incorporación de los estudios de las mujeres/perspectiva de género en los contenidos curriculares. Como fases del cambio curricular, menciona seis, la primera, la invisibilización de las mujeres, sería el grado cero desde el que se comienza a desarrollar la innovación curricular. En una segunda fase, la de búsqueda de mujeres ausentes, el objetivo principal sería la recuperación de mujeres notables de todos los campos a lo largo de la historia. En la tercera etapa, las mujeres son consideradas un grupo subordinado, cuya participación ha sido devaluada a lo largo de la historia. Una cuarta etapa estaría enfocada, antes que en las mujeres como grupo, en la crítica al androcentrismo. Una quinta fase, deudora de la anterior, estaría vinculada a la crítica de las disciplinas y su arsenal conceptual y metodológico. Por último, la sexta y última etapa la constituiría el currículum transformado, donde el interés se desplazaría a la intersección del género con la clase social y la raza, y la elaboración de conocimientos que den cuenta de la diversidad humana.
} 
manera transversal, particularizando contenidos y orientaciones para las distintas áreas. Se trataba, en definitiva, de "integrar la equidad de género entre los principios y valores fundamentales de la educación” (PRIOM, 1991: 17). En este sentido, podemos identificar dos núcleos de preocupaciones, que evidencian los intentos por transformar las articulaciones existentes entre género y trabajo, en sintonía con algunas de las cuestiones que eran abordadas en los manuales:

Por un lado, en varias áreas se insiste en identificar particularmente los aportes de las mujeres. Esto es señalado para el área artística y de filosofía, pero particularmente para las ciencias naturales (física y química) para revertir su invisibilización en las mismas. Se propone también incentivar a las mujeres de manera específica en esta área, y también en la de matemáticas y tecnología, para contrarrestar las expectativas de rendimiento de niños y niñas, que mantienen a las segundas alejadas de estas áreas.

Otro núcleo que aparece de manera recurrente es el análisis del estatus de las mujeres cómo grupo, y la valorización de sus aportes al desarrollo económico social. En el área de historia, se propone dar cuenta del papel de las mujeres en cada uno de los períodos históricos, de la transformación de su condición en la sociedad actual, y se añade para el polimodal, el estudio del movimiento social de mujeres y el feminismo. En el área de economía y geografía se propone analizar los aportes de la mujer a la economía nacional e internacional, su participación en el desarrollo y sus contribuciones a la agricultura, la industria y los servicios, y la feminización de la pobreza, así como también analizar la separación de la vida familiar y laboral, la división sexual del trabajo, y el valor económico del trabajo femenino en el hogar.

Cómo puede verse tanto en esta propuesta como en los materiales para la formación docente, a partir de un ejercicio crítico sobre las propias prenociones de las y los docentes y la provisión de herramientas para la reflexión o el desarrollo de actividades, el programa se propuso incidir en las prácticas de enseñanza que estos desarrollaban. Al colocar la constitución generizada del mercado de trabajo, la desigual distribución del trabajo doméstico y la propia configuración interna del trabajo docente como ejes de problematización, el PRIOM pretendió transformar las articulaciones entre género y trabajo, que marcaban este vínculo con la desigualdad.

Estos intentos de modificar la política educativa a partir de la incorporación de la perspectiva de género en la currícula y la formación docente desarrollados por el PRIOM en los 90, se vieron coartados por la resistencia de la jerarquía católica y del gobierno menemista. En sintonía con las posiciones que sostuvieron ambos actores en la reforma constitucional de 1994, en la Conferencia Internacional sobre Población y Desarrollo y la IV Conferencia internacional de la mujer se opusieron a incorporar el género, arguyendo que desnaturalizaba la necesaria complementariedad de los sexos, en el marco de la familia heterosexual reproductiva. Está resistencia llevo a que las integrantes del PRIOM renunciaran luego de que la primera versión de los $\mathrm{CBC}$, que incorporaba alguna de las modificaciones propuestas, fuera reemplazada por otra más en sintonía con los 
postulados católicos, echando por tierra los intentos de incorporar la perspectiva de género en educación.

\section{La calle, la academia, las políticas públicas. Flujos y reflujos en la configuración de políticas públicas}

Comenzábamos este escrito enmarcando nuestro trabajo en un proyecto colectivo de investigación que se plantea analizar la intervención estatal en la reproducción y transformación de desigualdades de género. Señalamos que uno de los objetivos de este proyecto es indagar cómo algunas dimensiones de los marcos interpretativos construidos por los feminismos, entendidos como "conjunto histórica y culturalmente específico de recursos discursivos de los que disponen los miembros de una colectividad social determinada para plantearse reivindicaciones unos a otros" (Fraser, 2015 [1989]: 79), han logrado permear el diseño de políticas públicas. En este marco, nos propusimos indagar cómo el trabajo fue construido como una dimensión fundamental de la desigualdad de género, y cómo algunos de los aportes teóricos que se desarrollaron a partir de este reconocimiento lograron permear el diseño de políticas públicas, en un contexto paradójico de expansión de derechos y consolidación del capitalismo en Argentina. Nos centramos especialmente en cómo este movimiento tuvo lugar en el marco de las políticas educativas, que se propusieron modificar las formas tradicionales de socialización de género en la escuela y alterar por este medio la configuración generizada de la organización económica y del trabajo.

En el primer apartado nos centramos en el proceso que llevó la problematización del trabajo de la lengua tartamuda de la calle a los claustros académicos, delineando los contornos de un marco interpretativo. De la mano de la movilización política y social que caracterizó los años 70, y en medio de un contexto autoritario, comenzaron a tejerse una serie de términos para problematizar la situación subordinada de la mujer. El trabajo doméstico fue uno de esos términos clave, que sirvió para resaltar el trabajo invisible de las mujeres al interior del hogar como necesario para la reproducción social, tal y como señalaron tempranamente Larguia y Dumoulin (Theumer, Bellucci, 2018). En las décadas siguientes, de la mano de la Economía Feminista estos planteos iniciales sirvieron de apoyatura para cuestionar la ciencia económica hegemónica y su concepción androcéntrica del trabajo, lo que derivó en una crítica al concepto acotado de trabajo de gran utilidad para analizar los procesos de segregación laboral por género y la valoración económica y social diferencial de los distintos trabajos y actividades.

En el segundo apartado buscamos mostrar cómo, en un contexto de reconocimiento de los derechos de las mujeres a escala internacional y de apertura democrática en la escala regional, se abrió un nuevo escenario que permitió que algunos aspectos de los marcos interpretativos delineados por el feminismo lograran cierto grado de institucionalidad. Con la llegada al Estado de activistas y expertas feministas se estableció un "puente" entre las demandas de los movimientos feministas y de mujeres por lo menos de aquellas que consideraban legítima la participación en el Estado- y el 
desarrollo de políticas públicas. Esto implicó la traducción de algunos de los desarrollos teóricos en torno al trabajo en propuestas de intervención concreta. La educación se conformó en un ámbito estratégico para intentar remover las marcas de género que la escuela (re) producía al desvalorizar al trabajo doméstico y socializar de forma diferencial a mujeres y varones reforzando actividades y roles generizados. A partir de propuestas de formación docente y de modificación curricular, se buscó permear el ámbito educativo un nuevo repertorio de recursos discursivos que permitieran problematizar, la vez que transformar, las formas en que género y trabajo se articulaban en el ámbito educativo. Si bien está experiencia quedó truncada por la reacción conservadora de sectores religiosos y su alianza con el gobierno menemista, serviría de antesala a los debates que se darían en los años 2000 sobre la inclusión curricular de la educación sexual integral.

Así, el recorrido que realizamos en este trabajo buscó mostrar el derrotero que llevó a algunas conceptualizaciones feministas y del movimiento de mujeres del ámbito de la calle, al de la académica y las políticas públicas. Lo hicimos con el objetivo de mostrar cómo cierto repertorio de recursos discursivos novedosos son construidos y se ponen a circular por los más diversos espacios, transformándose en cada interacción, pero habilitando -y también constriñendo- a las sujetas y sujetos a problematizar diversas aristas de la realidad social, a la vez que a ensayar soluciones a las mismas. La tarea que resta - y a la que estamos abocades desde el proyecto de investigación- es la de indagar las formas en que estos marcos interpretativos son apropiados, resistidos o ignorados por les agentes encargades de llevar a cabo la política y por sus destinataries.

\section{Bibliografía}

Anzorena, Claudia (2013a): Mujeres en la trama del Estado. Una lectura feminista de las políticas públicas. Mendoza: EDIUNC.

Anzorena, Claudia (2013b): "La institucionalización de la "cuestión de género" a 26 años de Nairobi: el segundo Instituto Provincial de la Mujer de Mendoza (2008-2011)", en La Aljaba, Volumen 27, p. 59-74

Arriagada, Irma (1997): "Realidades y mitos del trabajo femenino urbano en América Latina". En Series mujer y desarrollo. CEPAL, Santiago de Chile.

Arruza, Cinzia. (2015). Las sin partes. Matrimonios y divorcios entre feminismo y marxismo. Editorial Sylone.

Barrancos, Dora (2007): Mujeres en la sociedad argentina. Una historia de cinco siglos. Buenos Aires: Sudamericana.

Benería, Lourdes. (1999): “La aparición de la economía feminista”, en Historia Agraria, № 
Revista Punto Género № 12. Diciembre de 2019

ISSN 0719-0417 / 21 - 47

17. pp 59- 62.

Benston, Margaret (1972): "Para una economía política de la liberación femenina", en La liberación de la mujer: año cero, p. 33-47. Buenos Aires: Granica Editor.

Brosio, Magalí. (2016):“Introducción a la medición de la brecha salarial por género y sus determinantes". En Blog Economía Feminista. Octubre. Disponible en: http://economiafeminita.com/introduccion-a-la-medicion-de-la-brecha-salarial-por-generoy-sus-determinantes.

Brown, Josefína (2011). Mujeres y ciudadanía en Argentina. Debates teóricos y políticos sobre derechos (no) reproductivos y sexuales (1990-2006). Teseo.

Carrasco, Cristina (1997). "Mujeres, trabajos y políticas sociales en España. Una nueva dimensión del Estado del Bienestar”. DUODA Revista d'Estudis Feministes, № 13, pp. 85104.

Carrasco, Cristina. (2001): "La sostenibilidad de la vida humana: ¿Un asunto de mujeres?”. En: Mientras Tanto, № 82 pp. 43-70.

Carrasco, Cristina (2014). Economía, trabajos y sostenibilidad de la vida. En Jubeto Ruiz; Larrañaga Sarriegi; Carrasco Bengoa: León Trujillo; Herrero López; Salazar de la Torre; Ayuso; Salcedo Carrión y Pérez Alba (comps). Sostenibilidad de la vida. Aportaciones desde la economía solidaria, feminista y ecológica (pp. 27-41). Bilbao: REAS Euskadi.

Carrasco, Cristina; Galvéz Lina y Jubeto, Yolanda (2016): "Semimonográfico La economía feminista en diálogo con otras perspectivas críticas", en Revista de Economía crítica, № 22 , p. 88-91.

Costa, Ana Alice Alcántara (2011). "El movimiento feminista en Brasil: dinámicas de una intervención política”. En Anuario de Hojas de Warmi № 16.

Cutuli, Romina (2012): "Medir es conocer. Economía feminista y cuantificación del trabajo", en Observatorio laboral Revista Venezolana Universidad de Carabobo Venezuela. Volumen. 5, № 9, enero- junio pp. 23- 41.

Dalla Costa, Mariarosa. (2006): "La sostenibilidad de la reproducción: de las luchas por la renta a la salvaguarda de la vida" en: Transformaciones del trabajo desde una perspectiva feminista. Producción, reproducción, deseo, consumo, p.59-18. Madrid: Laboratorio feminista.

Dumoulin, John y Larguía, Isabel (1976) Hacia una ciencia de la liberación de la mujer. Coleccion Cuadernos ANAGRAMA. 
Ergas, Yasmine (2000 [1990]): "El sujeto mujer: el feminismo de los años sesentaochenta", en Duby, Georges y Perrot, Michelle (comp.) Historia de las mujeres en Occidente, p. 539-566. Madrid: Taurus.

Facio, Alda (2011). "Viena 1993, cuando las mujeres nos hicimos humanas". En Pensamiento iberoamericano, ISSN 0212-0208, №. 9.

Faur, Eleonor y Zamberlin, Nina (2008). "Gramáticas de género en el mundo laboral. Perspectivas de trabajadoras y trabajadores en cuatro ramas del sector productivo del área metropolitana de Buenos Aires". Cepal. Colección documentos de proyectos.

Federici, Silvia. (2013): Revolución en punto cero. Trabajo doméstico, reproducción y luchas feministas. España: Traficantes de Sueños.

Fraser, Nancy (2015 [1989]). Fortunas del feminismo. Del capitalismo gestionado por el estado a la crisis neoliberal, Traficantes de Sueños.

Giordano, Verónica (2012): "La celebración del Año Internacional de la Mujer en Argentina (1975): acciones y conflictos”, en Estudos Feministas, N²0, pp. 75-94.

Guzmán Stein, Laura y Pacheco Oreamuno, Gilda (1996): "La IV Conferencia Mundial sobre la Mujer: interrogantes, nudos y desafíos sobre el adelanto de las mujeres en un contexto de cambio", en Estudios Básicos de Derechos Humanos IV; p. 32. San José de Costa Rica: Ed. Instituto Interamericano de Derechos Humanos.

Hirata, Helena y Kergoat, Daniele. (1997). La división sexual del trabajo. Permanencia y cambio. Asociación Trabajo y Sociedad, Buenos Aires, Centro de Estudios de la Mujer, CEIL/PIETTE.

Hochschild, Arlie (2008): La mercantilización de la vida íntima. Buenos Aires: Katz.

Kessler, Gabriel (2014): Controversias sobre la desigualdad. Argentina 2003- 2013. Buenos Aires: FCE.

Lau, Ana. 2011. "El neo feminismo mexicano (1968-2010)". En labrys, études féministes/ estudos feministas, №19.

Liepitz, Alain (1996) "Género, clases y reparto del trabajo", en Rodríguez, Goñi y Maguregui (eds.) El futuro del trabajo. Reorganizar y repartir desde la perspectiva de las mujeres. Bilbao, Bakeaz

Méda, Dominique (1998). El trabajo. Un valor en peligro de extinción. Gedisa Editorial, Barcelona. 
Revista Punto Género № 12. Diciembre de 2019

ISSN 0719-0417 / 21 - 47

Morgade, Graciela (2001). Aprender a ser mujer, aprender a ser varón. Ediciones Novedades Educativas, Buenos Aires.

Nash, Mary (2012): Mujeres en el mundo. Historias, retos y movimientos. Madrid: Alianza.

Pecheny Mario (2014): Derechos humanos y sexualida: hacia la democratización de los vínculos afectivos en Argentina. En Sudamerica nํ3

Saporta, Nancy, Marysa Navarro, Patricia Chuchryk y Álvarez Sonia (1994). Feminismo en América Latina:De Bogotá a San Bernardo. En: M. León (comp.) Mujeres y Participación política. Avances y desafíos en América Latina. Bogotá. Mundo Editores

Rodríguez Enríquez y Pautassi (2014). La organización social del cuidado de niños: elementos para la construcción del cuidado en Argentina. Argentina: Equipo Latinoamericano de Justicia y Género.

Rodríguez Enríquez, Corina (2015): "Economía feminista y economía de cuidado. Aportes conceptuales para el estudio de la desigualdad". En: Revista Nueva Sociedad, № 256, pp. 30- 44.

Rodríguez Gustá, Ana Laura (2008): "Las políticas sensibles al género: variedades conceptuales y desafíos de intervención “. En: Temas y Debates, №16.

Tinsman, Heidi (2016). Se compraron el modelo Consumo, uva y la dinámica transnacional: Estados Unidos y Chile durante la Guerra Fría. Ediciones Universidad Alberto Hurtado, Santiago de Chile.

Theumer, Emmanuel y Bellucci, Mabel (2018). Desde la Cuba revolucionaria. Feminismo y marxismo en la obra de Isabel Larguía y John Dumoulin. Colección Grupos de Trabajo. CLACSO.

Thiesus Atschul, Monique (2015) "Zita C. Montes de Oca: la funcionaria y la feminista". En: Las conquistas de las mujeres en los 30 años de la democracia. Buenos Aires: APDH/INADI.

Todaro Rosalba, Abramo Lais y Godoy Lorena (2001): "Desempeño laboral de hombres y mujeres: opinan los empresarios". En: Cadernos Pagu (17/18), pp.197-236.

Trebisacce, Catalina (2016): "Una historia crítica del concepto de experiencia de la epistemología feminista", en Scielo ํ5 57 Diciembre.

Valdivia, Verónica (2010) '"¡Estamos en guerra señores!'. El régimen militar de Pinochet y el 'pueblo'. 1973-1980", en Historia 43:1, Santiago 
Revista Punto Género № 12. Diciembre de 2019

ISSN 0719-0417 / 21 - 47

Wainerman, C. \& Barck de Raijman, R. (1987). "Sexismo en los libros de lectura de la escuela primaria". Buenos Aires: IDES.

Wainerman, C. \& Heredia, M. (1999). ¿Mamá amasa la masa? Cien años de libros de lectura de la escuela primaria. Buenos Aires: Editorial de Belgrano.

Wainerman, Catalina (2005): La vida cotidiana en las nuevas familias: ¿una revolución estancada? Buenos Aires: Lumiere.

Wainerman, Catalina (1994): "Viviendo en familia: ayer y hoy". En Vivir en familia. Buenos Aires: Losada/UNICEF

\section{Fuentes}

La Convención sobre la Eliminación de Todas las Formas de Discriminación contra la Mujer (1979). Texto Accesible en: https://www.un.org/womenwatch/daw/cedaw/text/sconvention.htm

Ministerio de Cultura y Educación (1992). Programa Nacional de Promoción de la Igualdad de Oportunidades en el Área Educativa (PRIOM), Documento de Trabajo №1. Disponible En: http://www.bnm.me.gov.ar/giga1/documentos/EL004640.pdf

Ministerio de Cultura y Educación (1993). Educando a Mujeres y varones en el S.XXI. PRIOM. Disponible En: http://www.bnm.me.gov.ar/giga1/documentos/EL000252.pdf

Gloria Bonder y Gabriela Morgade (1993). Propuesta para Integrar los aportes de los Estudios de la Mujer a los Contenidos Básicos Curriculares, Ministerio de Cultura y Educación, PRIOM. Disponible En: http://repositorio.educacion.gov.ar:8080/dspace/handle/123456789/92330

Consejo Nacional de la Mujer (1994). Informe Nacional. Situación de la Mujer en la República Argentina. Disponible En: http://www.bnm.me.gov.ar/giga1/documentos/EL000258.pdf

Centro de Documentación de la Biblioteca Nacional de los maestros. Documento: La igualdad de oportunidades para varones y mujeres en el sistema educativo: una meta educativa (1993), PRIOM.

Poder Ejecutivo Nacional: Decreto No 659/74.

: Decreto № 2274/86

: Decreto $N^{\circ} 378 / 91$.

: Decreto No 1426/92. 